\title{
Evaluation of Pozzolanic Reactivity of Artificial Pozzolans
} J. Pontes ${ }^{1, a}$, A. Santos Silva ${ }^{2, b}$ and P. Faria ${ }^{1,0}$

'Department of Civil Engineering - Nova University of Lisbon, Caparica, Portugal ${ }^{2}$ National Laboratory of Civil Engineering, Av. Do Brasil 101, Lisbon, Portugal ajorgempontes@gmail.com, ’ssilva@Inec.pt, cpaulina.faria@fct.unl.pt

\begin{abstract}
Pozzolanicity is a very interesting issue regarding building materials, as a way to enhance mortars and concrete durability. This property results from the reaction between calcium hydroxide and silica and alumina based materials. Different types of natural and artificial pozzolans show pozzolanic activities that differ depending on the materials characteristics. Therefore, the study of this property, namely its reactivity with calcium hydroxide, reveals itself to be important in the selection of the type and content of these materials.

This paper presents the results of several pozzolanic reactivity methods, applied to different pozzolanic materials. The selected pozzolanic methods include Chapelle method, Fratinni method and Strength Activity Index. Those tests have been applied to evaluate the reactivity of various kinds of artificial pozzolans. The correlation between the test methods are presented and discussed.
\end{abstract}

Keywords: Pozzolan; Reactivity; Chapelle; Fratinni; Strenght Activity Index; fly ash; metakaolin 